\title{
ON FOURIER TRANSFORM MULTIPLIERS IN $L^{p}$
}

\author{
G. O. OKIKIOLU \\ (Received 29 April 1969; revised 9 April 1970) \\ Communicated by E. Strzelecki
}

\section{Introduction}

We denote by $R$ the set of real numbers, and by $R^{n}, n \geqq 2$, the Euclidean space of dimension $n$. Given any subset $E$ of $R^{n}, n \geqq 1$, we denote the characteristic function of $E$ by $\chi_{E}$, so that $\chi_{E}(x)=1$ if $x \in E$; and $\chi_{E}(x)=0$ if $x \in R^{m} \backslash E$. The space $L^{p}\left(R^{n}\right) \equiv L^{p}$ consists of those measurable functions $f$ on $R^{n}$ such that

$$
\left(\int_{R^{n}}|f(t)|^{p} d t\right)^{1 / p} \equiv\|f\|_{p}
$$

is finite. Also, $L^{\infty}$ represents the space of essentially bounded measurable functions with $\|f\|_{\infty}=\inf \{\alpha>0 ; m(\{x:|f(x)|>\alpha\})=0\}$, where $m$ represents the Lebesgue measure on $R^{n}$. The numbers $p$ and $p^{\prime}$ will be connected by $1 / p+1 / p^{\prime}=1$.

The Fourier transform of a function $f$ in $L^{1}$ is defined by

$$
\mathscr{F}(f)(x)=(2 \pi)^{-\frac{1}{2} n} \int_{R^{n}} f(t) e^{i x \cdot t} d t,
$$

where for $x=\left(x_{1}, x_{2}, \cdots, x_{n}\right), t=\left(t_{1}, t_{2}, \cdots, t_{n}\right)$, we set $x \cdot t=x_{1} t_{1}+$ $+x_{2} t_{2}+\cdots x_{n} t_{n}$.

The symbol $\mathscr{F}$ will also represent the extension of the Fourier operator as a bounded linear operator in $L^{p}, 1 \leqq p \leqq 2$.

Given a bounded measurable function $g$ on $R^{n}$ we denote by $T_{g}$ the operator defined on $L^{2}\left(R^{n}\right)$ by

$$
\mathscr{F} T_{g}(f)=g \mathscr{F}(f) ;\left(f \in L^{2}\right) .
$$

On using the fact that $\|\mathscr{F}(f)\|_{2}=\|f\|_{2}$ for $f$ in $L^{2}$, we see that

$$
\left\|T_{\theta}(f)\right\|_{2} \leqq\|g\|_{\infty}\|f\|_{2} \text {. }
$$

As usual we say that $g$ is a multiplier in $L^{p}, p \neq 2$, if $T_{g}$ can be extended to bounded linear operator in $L^{p}$. For properties of multipliers in $L^{p}$ see for example, Hörmander [2] and Brainerd and Edwards [1]. The main purpose of this paper is to describe some classes of bounded measurable functions on $R^{n}$ which are 
multipliers in $L^{p}, 1<p<\infty$. A particular case of the main theorem shows that some non-negative bounded symmetrically non-increasing function on $R$ are multipliers in $L^{p}(R), 1<p<\infty$. The conclusions obtained here supplement existing results on multipliers in $L^{p}$ spaces; see S. G. Mihlin [6], Hörmander [2, Theorem 2.5], Stein [7], de Leeuw [3], Littman [4], and also [5]. See also [10].

I am grateful to the referee of this paper for his comments.

\section{The main results}

The main results of the paper are given in Theorem 2.4 and Corollary 2.5 of this Section. In the next Section we consider the special results involving the one-dimensional cases. First we state two results which will be employed in the proof of the main theorem.

\subsection{Definitions}

(2.1.1) Given any measurable function $h$ on $R^{n}$ we define the set $H^{a}, a>0$, by

$$
H^{a}=\left\{x \in R^{n}:|h(x)|>a\right\} .
$$

(2.1.2) For any real-valued function $\phi$ on $R$ and a function $h$ on $R^{n}$, we denote by $\phi \circ h$ the function whose value at $x \in R^{n}$ is $\phi(h(x))$.

(2.1.3) We denote by $\mathscr{J}^{n}(n \geqq 1)$ the class of rectangles $I$ in $R^{n}$ of the form $I=I_{1} \times I_{2} \times \cdots \times I_{n}$, where each $I_{j}$ is an interval of $R$ (open, closed, or half open).

2.2. LemMA. Let $\phi$ be absolutely continuous on $[0, \infty)$, with $\phi(0)=0$, and let $h$ be measurable and non-negative on $R^{n}$. Then, with $\phi^{\prime}(x)=(d / d x) \phi(x)$,

$$
\phi \circ h(x)=\int_{0}^{\infty} \phi^{\prime}(a) \chi_{H^{a}}(x) d a, \quad\left(x \in R^{n}\right) .
$$

Proof. This follows immediately from the fact that

$$
\phi \circ h(x)=\int_{0}^{h(x)} \phi^{\prime}(a) d a .
$$

2.3. Lemma. If $1<p<\infty, n \geqq 1$, then there is a constant $k_{p, n}$ such that, for $J \in \mathscr{J}^{n}$,

$$
\left\|T_{x_{J}}(f)\right\|_{p} \leqq k_{p, n}\|f\|_{p} .
$$

Proof. This is a well-known result which follows easily from the one-dimensional case. Note that if $J=[a, b) \subset R$, then

$$
T_{x_{I}}(f)(x)=\pi^{-1} \int_{R}\left(e^{-i a(t-x)}-e^{-i b(t-x)}\right)(t-x)^{-1} f(t) d t,
$$

and the boundedness of $T_{x_{I}}$ follows by applying known results involving the Hilbert transform [8, Theorem 101]. 
(2.3.1) NoTE. If $M=\bigcup_{k=\imath}^{j} I_{k}$, where $\left\{I_{1}, I_{2}, \cdots, I_{j}\right\}$ is a finite disjoint subclass of $\mathscr{J}^{n}$, then we have

so that by 2.3 ,

$$
T_{x_{M}}=\sum_{k=1}^{j} T_{x_{I_{k}}}
$$

$$
\left\|T x_{M}(f)\right\|_{p} \leqq j k_{p, n}\|f\|_{p}, \quad\left(f \in L^{p}\left(R^{n}\right)\right) .
$$

2.4. THEOREM. Let the functions $\phi$ on $[0, \infty)$ and $h$ on $R^{n}$ satisfy the following conditions:

(i) $\phi$ is absolutely continuous on $[0, \infty), \phi(0)=0$, and $\phi^{\prime} \in L^{1}(0, \infty)$,

(ii) $h$ is non-negative on $R^{n}$, and there is an integer $j \geqq 1$ such that, for $a>0$, we have

$$
H^{a}=\bigcup_{k=1}^{j(a)} H(a, k)
$$

where $j(a) \leqq j$ or $H^{a}=\emptyset$, each set $H(a, k) \in \mathscr{J}^{n}$, and $H(a, k) \cap H(a, l)=\emptyset$ if $k \neq l$. Then for $1<p<\infty$ and with $k_{p, n}$ defined as in Lemma 2.3; we have

$$
\left\|T_{\phi \circ h}(f)\right\|_{p} \leqq j k_{p, n}\left\|\phi^{\prime}\right\|_{1}\|f\|_{p} .
$$

Proof. Let $f \in L^{1} \cap L^{\infty}$. Then by Lemma 2.2, we have

$$
\mathscr{F} T_{\phi \circ h}(f)(x)=\int_{0}^{\infty} \phi^{\prime}(a) \chi_{H^{a}}(x) \mathscr{F}(f)(x) d a=\int_{0}^{\infty} \phi^{\prime}(a) \mathscr{F} T_{\chi_{H^{a}}}(f)(x) d a,
$$

and it follows by applying Fubini's theorem and the Parseval relation for Fourier transforms that, for $g \in L^{2}$,

$$
\begin{aligned}
\int_{R^{n}} g(x) \mathscr{F} T_{\phi \circ h}(f)(x) d x=\int_{0}^{\infty} \phi^{\prime}(a) \int_{R^{n}} \mathscr{F}(g)(x) T_{\chi_{H^{a}}}(f)(x) d x d a \\
=\int_{R^{n}} \mathscr{F}(g)(x) \int_{R^{n}} \phi^{\prime}(a) T_{\chi_{H^{a}}}(f)(x) d a d x .
\end{aligned}
$$

Further, on applying the Parseval relation once more and noting that the function $g$ is arbitrary, we see that

$$
T_{\phi \circ h}(f)(x)=\int_{0}^{\infty} \phi^{\prime}(a) T_{\chi_{H^{a}}}(f)(x) d a .
$$

Hence it follows from the hypotheses and Note 2.3.1, and by applying Minkowski's integral inequality that

$$
\left\|T_{\phi \circ h}(f)\right\|_{p} \leqq \int_{0}^{\infty}\left|\phi^{\prime}(a)\right|\left\|T_{\chi_{H^{a}}}(f)\right\|_{p} d a \leqq j k_{p, n}\left\|\phi^{\prime}\right\|_{1}\|f\|_{p} .
$$

The inequality can be extended to all of $L^{p}$ in the usual way by constructing sequences of functions in $L^{2} \cap L^{p}$ which converge to given functions in $L^{p}$-norm. 
2.5. COROllary. Let the function $h$ on $R^{n}, n \geqq 1$, be bounded and satisfy condition (ii) of Theorem 2.4. Then, for $1<p<\infty$ and with $k_{p, n}$ defined as in Lemma 2.3 , we have

$$
\left\|T_{h}(f)\right\|_{p} \leqq j k_{p, n}\|h\|_{\infty}\|f\|_{p} .
$$

Proof. Let $\phi_{0}$ be any monotone increasing absolutely continuous function on $[0, \infty)$ with $\left\|\phi_{0}^{\prime}\right\|_{1}=1$ and $\phi_{0}(0)=0$; for example we may choose

$$
\phi_{0}(t)=1-e^{-t} \text {. }
$$

Now define the function $\phi(x)$ by $\phi(x)=(1+\varepsilon)\|h\|_{\infty} \phi_{0}(x)$, where $\varepsilon>0$. We denote by $\phi^{-1}$ the inverse of $\phi$, so that for $y \in\left[0,\|h\|_{\infty}\right]$,

$$
\phi \circ \phi^{-1}(y)=y \text {. }
$$

Since $\left\{x \in R^{n}: \phi^{-1} \circ h(x)>a\right\}=\left\{x \in R^{n}: h(x)>\phi(a)\right\}$ it follows that if $h(x)$ satisfies the condition (ii) of Theorem 2.4, then so also does $\phi^{-1} \circ h(x)$. Hence by Theorem 2.4, we have, for $1<p<\infty$,

$$
\left\|T_{h}(f)\right\|_{p}=\left\|T_{\phi \circ \phi-10 h}(f)\right\|_{p} \leqq j k_{p, n}\left\|\phi^{\prime}\right\|_{1}\|f\|_{p}=(1+\varepsilon) j k_{p, n}\|h\|_{\infty}\|f\|_{p},
$$

and the required conclusion follows since $\varepsilon$ is arbitrary.

(2.5.1) Remark. It is possible to prove Corollary 2.5 directly by using the same argument as that given in the proof of Theorem 2.4 . We simply note that, as in Lemma 2.2,

$$
h(x)=\int_{0}^{h(x)} d a=\int_{0}^{b} \chi_{H^{a}}(x) d a,
$$

where $b=(1+\varepsilon)\|h\|_{\infty}, \varepsilon>0$.

\section{The one-dimensional case of the main result}

The main feature of the one-dimensional case of Theorem 2.4 is that condition (ii) of that theorem can be put in a more suitable form involving in some cases the monotone-ness of the function $h$.

3.1. THEOREM. Let the function $\phi$ on $[0, \infty)$ and $h$ on $R$ satisfy the following conditions

(i) $\phi$ is absolutely continuous on $[0, \infty), \phi(0)=0$, and $\phi^{\prime} \in L^{1}(0, \infty)$,

(ii) $h$ is non-negative on $R$, and there is a finite class $\left\{A_{1}, A_{2}, \cdots, A_{j}\right\}$ of disjoint intervals such that

$$
R=\bigcup_{k=1}^{j} A_{k}
$$

where $h$ is monotone on each set $A_{k}$. Then for $1<p<\infty$, there is a constant $k_{p}$ such that

$$
\left\|T_{\phi \circ h}(f)\right\|_{p} \leqq j k_{p}\left\|\phi^{\prime}\right\|_{1}\|f\|_{p} .
$$


Proof. This result is a special case of Theorem 2.4. We note that, for $a>0$, we have

$$
H^{a}=\{x \in R: h(x)>a\}=\bigcup_{k=1}^{j} H(a, k),
$$

where $H(a, k)=\left\{x \in A_{k}: h(x)>a\right\}$. Since $h$ is monotone on the interval $A_{k}$, the set $H(a, k) \in \mathscr{J}^{1}$, and so condition (ii) of Theorem 2.4 is satisfied.

3.2. COROLLARY. Let the function $h$ on $R$ be bounded and satisfy the condition (ii) of Theorem 3.1. Then for $1<p<\infty$ there is a constant $k_{p}$ such that

$$
\left\|T_{h}(f)\right\|_{p} \leqq j k_{p}\|h\|_{\infty}\|f\|_{p} .
$$

Proof. In view of the remarks in the proof of Theorem 3.1, the result is easily seen to be a special case of Corollary 2.5 .

3.3. Remarks. (i) The conclusions of Corollary 3.2 provide examples of multipliers in $L^{p}(R), 1<p<\infty$, which have an infinite set of discontinuities on some subinterval of $R$. The main condition required of such functions is that they should be non-increasing or non-decreasing on the interval. (ii) Since linear combinations of multipliers are also multipliers the condition in Corollary 3.2 that $h$ be non-negative can be replaced by one requiring that $h$ be a linear combination of functions satisfying the conditions given there. For this reason the conclusions of the Corollary apply to functions of the form

$$
h(t)=\int_{0}^{t} g(\tilde{\zeta}) d \xi
$$

where the integral is bounded on $R$.

\section{References}

[1] B. Brainerd and R. E. Edwards, 'Linear operators which commute with translations', $J$. Austral. Math. Soc. 6 (1966) 289-327, 328-350.

[2] L. Hörmander, 'Estimates for translation invariant operators in $L^{p}$ spaces', Acta Math. 104 (1960), 93-140.

[3] K. de Leeuw, 'On $L^{p}$ multipliers', Ann. of Math. 81 (1965), 364-379.

[4] W. Littman, 'Multipliers in $L^{p}$ and interpolation' Bull. Amer. Math. Soc., 71 (1965), 764-766.

[5] W. Littman, C. McCarthy and N. Riviere, ' $L^{p}$-multiplier theorems', Studia Math. 30 (1968), 193-217.

[6] S. G. Mihlin, 'On the multipliers of Fourier integrals', Dokl. Akad. Nauk SSSR (N.S.) 109 (1956), 701-703.

[7] E. M. Stein, 'On limits of sequences of operators', Ann. of Math. 74 (1961), 140-170.

[8] E. C. Titchmarsh, Introduction to the theory of Fourier integrals, (Oxford, 1937).

[9] A. Zygmund, Trigonometric Series, Vols. I \& II (Cambridge, 1959).

Added in proof.

[10] G. O. Okikiolu, 'On the theory and applications of Dirichlet projections', J. für die reine angew. Math. 245 (1970), 149-164.

University of East Anglia

Norwich, England 